\title{
Prediction of quality attributes and ripeness classification of bananas using optical properties
}

\begin{abstract}
Consumers consider the ripeness of fruit as a very important factor in making a choice at the time of purchase. Ripeness in fruit generally affects the eating quality and market price of fruit. This study investigated the potential of using the optical properties of banana such as absorption, reduced scattering and effective attenuation coefficients extracted from backscattered images captured at five different wavelengths of 532,660,785, 830, and $1060 \mathrm{~nm}$ for predicting the quality attributes of the fruits. It was observed that there was a very strong correlation between the optical properties investigated and the banana ripening stages at wavelengths 532, 660 and $785 \mathrm{~nm}$. Absorption and effective attenuation coefficients showed a negative correlation with ripening stages while the reduced scattering coefficient exhibited a positive correlation with ripening stages. Prediction and classification models were developed using an artificial neural network to build both prediction and classification models. The visible wavelength region of 532, 660 and $785 \mathrm{~nm}$ gave the highest correlation coefficient $(\mathrm{R})$ range of $0.9768-0.9807$ for chlorophyll prediction and $0.9553-0.9759$ for elasticity prediction, while the near infrared region of 830 and $1060 \mathrm{~nm}$ gave an R range of 0.9640-0.9801 for prediction of the soluble solids content (SSC) when the absorption and reduced scattering coefficients were used. For the classification of banana into ripening stages $2-7$, the visible wavelength region gave the highest classification accuracy of $97.53 \%$. This study has shown that the optical properties of banana can be employed for nondestructive prediction and classification of banana into different ripening stages.
\end{abstract}

Keyword: Absorption; Light-tissue interaction; Non-invasive test; Scattering 